\title{
Design of Intelligent Controller for Chaotic Permanent Synchronous Motor
}

\author{
Negar Etemadi \\ Department of Electrical Engineering, \\ Islamic Azad University, \\ Gonabad Branch, Iran
}

\author{
Assef Zare \\ Department of Electrical Engineering, \\ Islamic Azad University, \\ Gonabad Branch, Iran
}

\begin{abstract}
In this paper, an intelligent controller design method for the chaotic permanent magnet synchronous motor stability is presented. Active control strategy is a powerful control technique in stability chaotic systems. Learning algorithm using active control techniques, and then intelligent controller will be used. The proposed method can reduce the dimensions of the controller. A comparative study has been one with active and adaptive neural fuzzy controller. Simulation results show that the proposed controller can be chaotic permanent magnet synchronous motor will converge to the unstable equilibrium points. The controller can zero error, while has been tracking well desired value.
\end{abstract}

\section{Keywords}

Neural fuzzy, chaotic, permanent magnet motor, synchronization

\section{INTRODUCTION}

The study of nonlinear systems which exhibit complex dynamic phenomena such as bifurcation and chaos has produced in the last few years a large number of contributions from all the areas of nonlinear science. Most of the research that has been developed is essentially related to aspect of the analysis such complex systems [1-3]. The control objectives are in chaos: the stability of chaotic behavior and remove it, synchronization of chaotic systems. The idea of controlling chaos was first introduced by Ott et al. [4], after them, variety of approach have been proposed for chaos control, such as: adaptive [5], fuzzy [6], sliding mode [7], impulsive [8], backstepping [9], and many more in [10].

Basically, the chaos synchronization problem means making two systems oscillate in a synchronized manner. Given a chaotic system, which is considered as the master system, and another identical system, which is considered as the slave system, the dynamical behaviors of these two systems may be identical after a transient time when the slave system is driven by a control input. Most publications of chaos synchronization often assume that the chaos synchronization systems are identical and the bounds of the external disturbances should be known in advance. Ever since the synchronization of chaotic dynamical systems has been observed by Pecora and Carroll in 1990 [11], chaos synchronization has become a topic of great interest [12-15].

The permanent magnet synchronous motors (PMSMs), having the advantages of high efficiency, high power density and fast dynamic response, are very suitable as the propulsion motor in compact electric propulsion systems. In the PMSM vector control or direct torque control systems, it requires the information of rotor speed for closed-loop control, so the position sensors such as an optical encoder or a resolver are needed to be installed on the shaft [16]. When system parameters falling into a certain area or under some working conditions, the permanent magnet synchronous motor (PMSM) will appear chaos phenomenon, which threatens the secure and stable operation of drive system. The PMSM was shown to exhibit chaos by Li and Park et al. in [17]. Some theories and control techniques have been applied to control chaos in PMSM. Ge and Huang developed an adaptive backstepping control approach for the PMSM in [18]; and the proposed controller stabilizes the chaos exhibited by the PMSM and drives it to the desired speed set-point. A feedback linearization control technique was implemented on the PMSM by Ren and Liu [19] to stabilize the system about a user determined set-point which is a point within the strange attractor of the PMSM. Furthermore, the method of time delay feedback control was successfully implemented by Ren et al. [20] to control the PMSM. The sliding mode control was addressed to control chaos for PMSM in [21]. And Wei et al. [22] proposed an adaptive dynamic surface control of chaos in PMSM. An instantaneous Lyapunov exponent control algorithm was used by Zribi et al. [23] to control the PMSM. However, the existing control methods also have some disadvantages. In this paper, an adaptive neural fuzzy control method is developed to suppress chaos in permanent magnet synchronous motor. To train an intelligent controller should choose a method of training. In this paper, active control method is used for training, and then the data will be used for training the neural fuzzy controller, while dimensions of the proposed intelligent controller are reduced. Simulation results show that the intelligent controller can stabilize the PMSM chaos.

The paper is organized as follows. After this introduction, section 2 focuses on the description of the chaotic PMSM system and mathematical mode. Section 3 discusses the design of the intelligent controller for suppressing chaotic motion as well as the control of chaos in the PMSM system. Finally, simulation results are presented in section 4 in order to shown method effectiveness. In section 5 conclusions is presented

\section{DYNAMIC OF PMSM AND MATHEMATIC ANALYSIS}

The mathematical model of the permanent magnet synchronous motor with smooth air-gap is described as follow [17].

$$
\begin{aligned}
& \frac{d i_{d}}{d t}=\frac{-R_{1} i_{d}+\omega L_{q} i_{q}+u_{d}}{L_{d}} \\
& \frac{d i_{q}}{d t}=\frac{-R_{1} i_{q}+\omega L_{q} i_{q}+u_{q}-\omega \psi_{r}}{L_{q}} \\
& \frac{d \omega}{d t}=\frac{\left.n_{p} \psi_{r} i_{q}+n_{p}\left(L_{d}-L_{q}\right) i_{q} i_{d}-T_{L}-\beta \omega\right)}{J}
\end{aligned}
$$


Where $i_{d}, i_{q}$ and $\omega$ are the state variable, which represent currents and motor angular frequency, respectively. $u_{d}$ And $u_{q}$ the direct and quadrature-axis stator voltage components, respectively. $J$ the polar moment of inertia, $T_{L}$ the external load torque, $\beta$ the viscous damping coefficient, $R_{1}$ the stator winding resistance, $L_{d}$ and $L_{q}$ the direct and quadrature axis stator inductors, respectively, $\psi_{r}$ the permanent magnet flux, and $n_{p}$ the number of pole - pairs. For clarity, this normalization is presented in the paper. Define the time scale $\tau$ to be such that $\tau=\frac{L}{R}$ and let the normalized time $\mathrm{t}$ to be such that $t=\frac{\bar{t}}{\tau}$. Also let the scalar $\kappa$ be such that $\kappa=\frac{\beta}{\tau n_{p} \psi_{r}}$. We will define the normalized states variables $i_{d}, i_{q}$ and $\omega=\tau \bar{\omega}$ such that:

$i_{d}=\frac{1}{\kappa} \bar{i}_{d}, \quad i_{q}=\frac{1}{\kappa} \bar{i}_{q}, \quad \omega=\tau \bar{\omega}$

In addition, we will define the normalized voltages $u_{d}$ and $u_{q}$ such that:

$u_{d}=\frac{1}{\kappa R} \bar{u}_{d}, \quad u_{q}=\frac{1}{\kappa R} \bar{u}_{q}$

The normalized load torque $T_{L}$ is such that $T_{L}=\frac{\tau^{2} T_{L}}{J}$. The constants parameters $\sigma$ and $\gamma$ are taken to be such that $\sigma=\frac{\beta \tau}{J}$ and $\gamma=\frac{-\psi_{r}}{\kappa L}$. With the PMSM model given by

Eq. 1 and using Eq.2 and Eq.3, one obtains the following normalized model of the PMSM [19]:

$$
\begin{aligned}
& \frac{d i_{d}}{d t}=-i_{d}+\omega i_{q}+u_{d} \\
& \frac{d i_{q}}{d t}=-i_{q}+\omega i_{d}+\gamma \omega+u_{q} \\
& \frac{d \omega}{d t}=\sigma\left(i_{q}-\omega\right)+T_{L}
\end{aligned}
$$

Where for the sake of simplicity, in this brief, we only study the dynamic characteristics of the smooth air gap PMSM, namely, $L_{q}=L_{d}=L$ in the model. Therefore, $i_{d}, i_{q}$ and $\omega$ are the state variable, which represent d-q axis currents and motor angular frequency, and $u_{d}, u_{q}$ are the d-q axis stator voltage. $T_{L}$ Denote the load torque, $\sigma, \gamma$ are system parameters. When system parameters falling into a certain area or under some working conditions, the permanent magnet synchronous motor will appear chaos phenomena. If the system parameters $\sigma=5,45, \gamma=20 \quad$ and $u_{d}=0, u_{q}=0, \mathrm{~T}_{L}=0$. For example, a typical chaotic attractor is shown in Fig.1 and Fig.2 are the time response of the state variable under chaos.

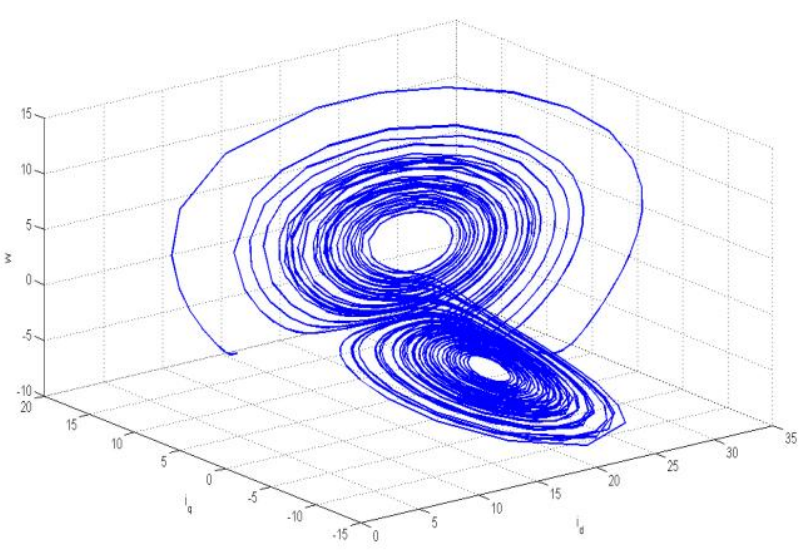

Fig. 1. The Chaotic Attractor of PMSM

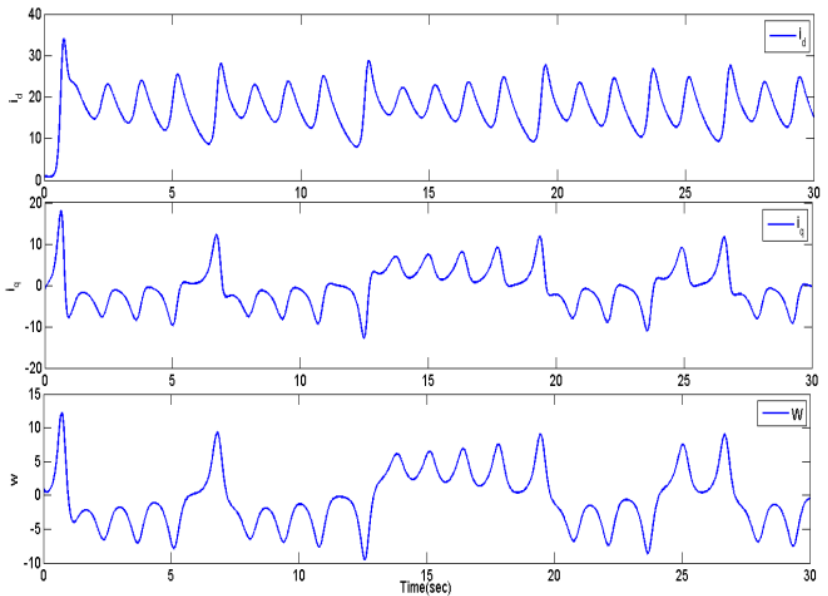

Fig. 2. The state curves without $u_{d}, u_{q}, \mathrm{~T}_{L}$

\section{INTELLIGENT CONTROLLER}

\subsection{Adaptive neural fuzzy}

The advantage of the fuzzy inference system is that it can deal with linguistic expressions and the advantage of a neural network is that it can be trained and so can self-learn and selfimprove. Jang (1993) took both advantages, combining the two techniques, and proposed the Adaptive Neuro-Fuzzy Inference System (ANFIS) [24]. The idea behind neural network and fuzzy inference combination is to design a system that uses a fuzzy system to represent knowledge in an interpretable manner and has the learning ability derived from a neural network that can adjust the membership functions parameters and linguistic rules directly from data in order to enhance the system performance ANFIS is a hybrid intelligent system which implements a Sugeno fuzzy inference system for a systematic approach to generating fuzzy rules from a given input output data set [25].

\subsection{Learning of controller}

In this section the method proposed in [5], the problem of will be studied chaotic system stability. We consider the chaotic system in form of: 
$\dot{X}=A x+f(x)$

Where $x \in R^{n}$ is the state vector, $A \in R^{n}$ is a constant system matrix, and $f: R^{n} \rightarrow R^{n}$ is a nonlinear sequence function. For the solving control problem, first define the error such as:

$e_{i}=x_{i}-x_{i d} \quad i=1,2,3$

Let $x_{i d},(i=1,2,3)$ denotes the desire point or an equilibrium point. The aim of is to controller that stabilizing chaotic system (5) and guarantees of the chaos suppression. So, the dynamic error equation of system can be expressed by Eq. 7

Trajectory error sense that $\lim \left|e_{i}(\mathrm{t})\right|=0$, therefore, the define controller:

$u_{i}=-F(x)+\varepsilon_{i} e_{i} \quad i=1,2,3$

Here, $\varepsilon$ is a gain controller and $-F(x)=f(x)$ is nonlinear feedback. Substituting Eq. 8 into Eq. 7, the error dynamic simplifies to:

$\dot{e}_{i}=\lambda_{i} e_{i} \quad i=1,2,3$

Theorem1: According to the proposed controller in Eq.8, PMSM converge to desire points (in limited time) will be.

Proof. : We take candidate Lyapunov function:

$V(e)=\frac{1}{2} \sum_{i=1}^{3} e_{i}^{2}$

This is a positive definite function on $\square^{n}$. By differentiating $\mathrm{V}$ along the trajectories of Eq. 9 we have:

$$
\begin{aligned}
& \dot{V}(e)=e_{1} \dot{e}_{1}+e_{2} \dot{e}_{2}+e_{3} \dot{e}_{3} \\
& \Rightarrow e_{1}\left(\varepsilon_{1} e_{1}\right)+e_{2}\left(\varepsilon_{2} e_{2}\right)+e_{3}\left(\varepsilon_{3} e_{3}\right) \\
& \Rightarrow\left(\varepsilon_{1} e_{1}^{2}+\varepsilon_{2} e_{2}^{2}+\varepsilon_{3} e_{3}^{2}\right)<0
\end{aligned}
$$

If $\varepsilon_{i},(i=1,2,3)<0$ the selected, then the error state vectors exponentially converge to zero. To implement this method, we assume that in Eq. $4 u_{d}=u_{q}=T_{L}=0$ and control for the Eq. 4 will be added.

$$
\begin{aligned}
& \frac{d i_{d}}{d t}=-i_{d}+\omega i_{q}+u_{1} \\
& \frac{d i_{q}}{d t}=-i_{q}+\omega i_{d}+\gamma \omega+u_{2} \\
& \frac{d \omega}{d t}=\sigma\left(i_{q}-\omega\right)+u_{3}
\end{aligned}
$$

Initial values of the chaotic PMSM is $\left[\begin{array}{lll}i_{d 0} & i_{q 0} & \omega_{0}\end{array}\right]^{T}=\left[\begin{array}{lll}2 & 3 & 5\end{array}\right]^{T}$ and system parameters is $\sigma=5.45, \gamma=20$ and controller gains are $\left[\begin{array}{lll}\varepsilon_{1} & \varepsilon_{2} & \varepsilon_{3}\end{array}\right]^{T}=-\left[\begin{array}{lll}1 & 1 & 1\end{array}\right]^{T}$. Fig. 3 show that active controller is applied for the PMSM.

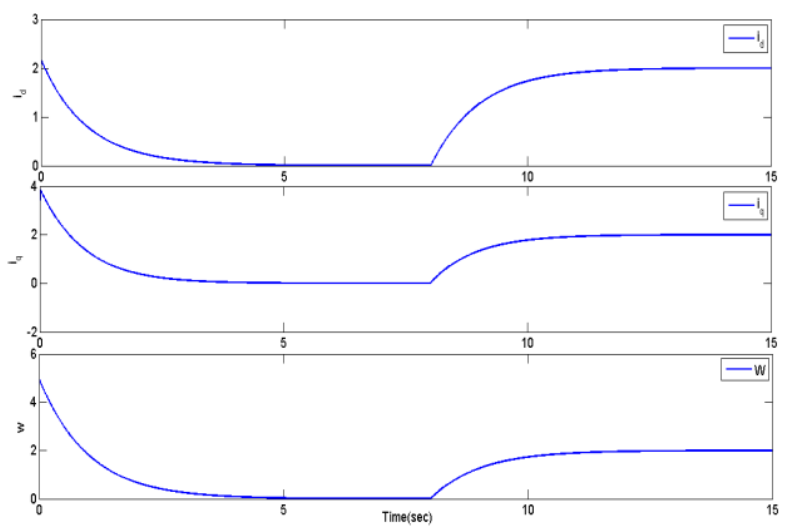

Fig.3 Active controller

The controller used from the beginning $(\mathrm{t}=0)$ and at the time $t=8$ seconds after removing the chaos has guided to a desired value.

\subsection{Intelligent Controller Structure}

The acronym ANFIS derives its name from adaptive neurofuzzy inference system. Adaptive fuzzy controller is designed for systems where high uncertainty, structure and system parameters are unknown. Generally, the main goals of adaptive controllers stabilize the system performance in the presence of uncertainty. The advanced fuzzy control should be adaptive.

The main idea of the technique, using an adaptive neural network to optimize the structure is fuzzy. This technique is a method for the fuzzy modeling process of to prepare the training data set. Thus, the membership function parameters in the fuzzy inference system so that we can obtain to best relationship. However, the data controller and error, learning intelligent controller can be done. As can be seen, the only intelligent controller will be added to all rows of equations. We assume that the controlled system is as follows:

$$
\begin{aligned}
& \frac{d i_{d}}{d t}=-i_{d}+\omega i_{q}+u_{1 A N F I S} \\
& \frac{d i_{q}}{d t}=-i_{q}+\omega i_{d}+\gamma \omega+u_{2 A N F I S} \\
& \frac{d \omega}{d t}=\sigma\left(i_{q}-\omega\right)+u_{3 A N F I S}
\end{aligned}
$$

Intelligent controller block diagram is depicted in Fig.4. In fact, learning is based on two parameters error and derivative.

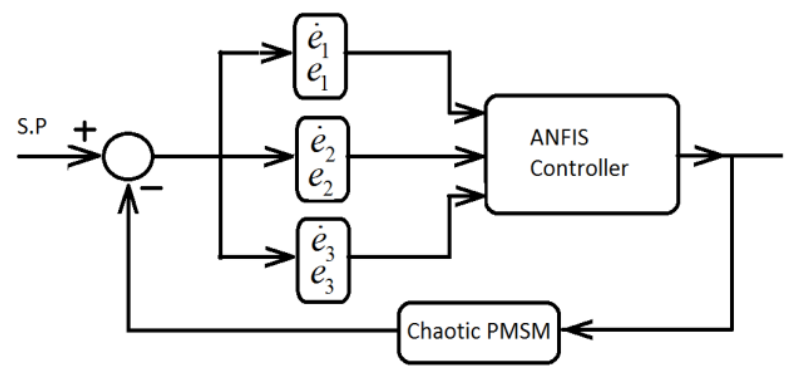

Fig.4 The block diagram proposed controller 
Table 1. Table architecture of ANFIS

\begin{tabular}{|l|l|l|}
\hline 1 & Type & Sugeno \\
\hline 2 & Imp Method & Prod \\
\hline 3 & Agg Method & Max \\
\hline 4 & Num Input & 2 \\
\hline 5 & Num Output & 1 \\
\hline 6 & Num Rule & 25 \\
\hline 7 & Epoch & $\mathbf{3 0}$ \\
\hline 8 & Num Mfs & $\mathbf{5}$ \\
\hline 9 & Mfs Type Input & Gauss2mf \\
\hline 10 & Mfs Type Output & Gauss2mf \\
\hline
\end{tabular}

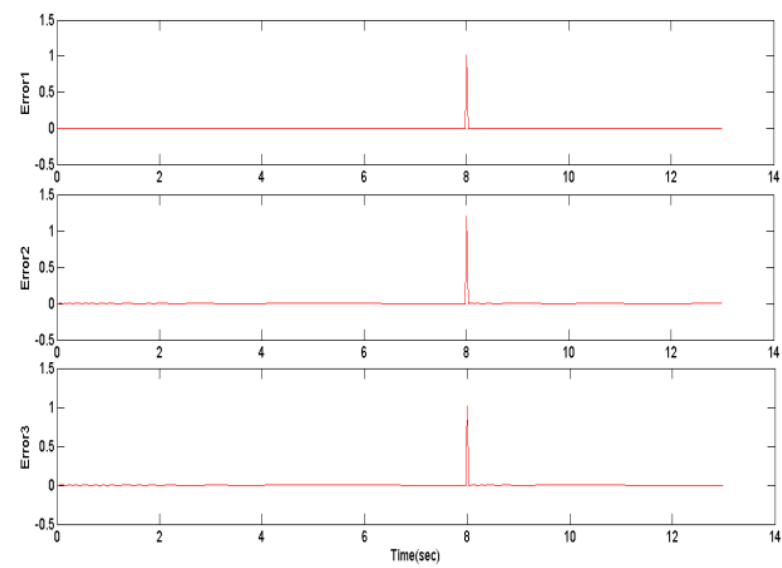

Fig. 5 train error of ANFIS controller

Figure 5 shows that repeating training error (Epoch) may be enough, and in Fig. 6 has been shown that active control (for training) can be stable permanent magnet synchronous motor and chaotic.

\section{NUMERIC SIMULATION}

According to equation (13) Output block diagram (3) has three controller. Each controller are set via table .1. As mentioned earlier, one of the properties of chaotic systems is sensitive to initial conditions. So, if a change in the initial conditions, the controller will be faced with a new behavior. Thus, the controller can be tested with different initial conditions. The initial conditions for this section are $\left[\begin{array}{lll}i_{d 0} & i_{q 0} & \omega_{0}\end{array}\right]^{T}=\left[\begin{array}{lll}2 & 4 & 6\end{array}\right]^{T}$ and system parameters is $\sigma=5.45, \gamma=20$.

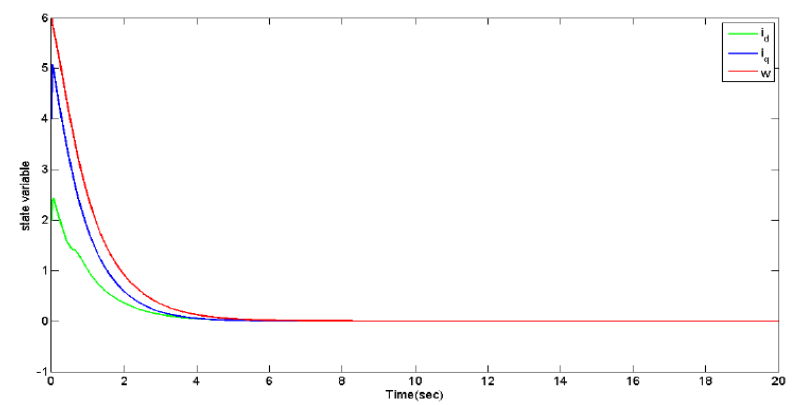

Fig.6 the behavior of PMSM controled

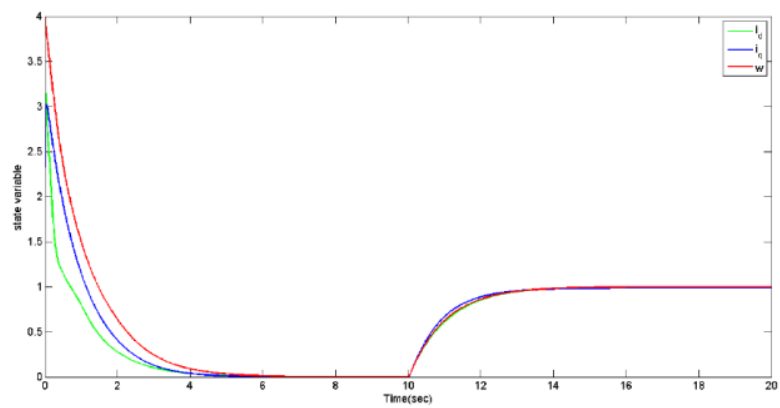

Fig.7 The behavior of chaotic PMSM controlled

According to the Fig.6, intelligent controller from $t=0$ seconds is applied to the motor. Almost at $t=4$ seconds of chaos has been removed. Again remember that is different initial conditions (with training). But in the Fig.7, we want to consider a desired value. In this way we can evaluate the performance of the controller. Here, too, the initial conditions are different. The controller tracking the desired value in less than $\mathrm{t}=3$ seconds .

\section{CONCLUSION}

In this paper, was investigated an intelligent control of permanent magnet synchronous motors. It was learning process using active control. In fact, by solving the active control answer, the correct answer is given learning process. Intelligent controller in simulation results were evaluated with different initial conditions, good results were obtained. In the second simulation, was considered a set point. The simulation results show that the essence of chaos is learning well. The architecture proposed in Table 1 is expressed almost the best mode available. Membership function of input-output type, number of repetitions, the most important factors. Intelligent controller must see PMSM workspace. For this reason in Fig. 6 after stability (suppression chaos) it is considered to be a desired value. It can be intelligent controller helps to understand the nature of chaotic PMSM. The simulation results show a good performance intelligent control.

\section{REFERENCES}

[1] T. Matsumoto, L. O. Chua and M. Komuro, (1985). The double scroll. IEEE Trans. Circuit syst, vol 32, p. 797-818

[2] J. P. Eckmann, and D. Rulle, (1985) Ergodic theory of chaos and strange attractors, Rev. Mod. Phys, vol. 75, no. 3 , p. $617-656$

[3] L. O. Chua, M. Komuro and T. Matsumoto, (1986) The double scroll family, IEEE Trans. Circuit Syst, vol. 33, p. 1072-118

[4] Ott E, Grebogi C, Yorke JA. (1990), Controlling Chaos. Phys Rev Lett; vol. 64, p. 1196-9

[5] Du Qu Wei, Xiao Shu Luo, (2007), "Passivity-based adaptive control of chaotic oscillations in power system", Chaos, Solitons and Fractals, vol. 31 p. 665671 ,

[6] Radu-Emil Precup • Marius L. Tomescu, (2014),Stable fuzzy logic control of a general class of chaotic systems, Neural Comput \& Applic, DOI 10.1007/s00521-014-1644-7

[7] Huaqing Li n, XiaofengLiao,ChuandongLi,ChaojieLi, (2011), Chaos control and synchronization via a novel 
chatter free sliding mode control strategy, Neurocomputing, vol. 74, p. 3212-3222

[8] Tao Yang, (Member IEEE), and Leon O. Chua, Fellow, (1997), Impulsive Stabilization for Control and Synchronization of Chaotic Systems: Theory and Application to Secure Communication, IEEE TRANSACTIONS ON CIRCUITS AND SYSTEMS-I: FUNDAMENTAL THEORY AND APPLICATIONS, vol. 44 , NO. 10 ,

[9] M.T. Yassen, (2006), Chaos control of chaotic dynamical systems using backstepping design, Chaos, Solitons and Fractals, vol. 27,p. 537-548

[10] S. BOCCALETTI, C. GREBOGI, Y.-C. LAI, H. MANCINI, D. MAZA, (2000),HE CONTROL OF CHAOS: THEORY AND APPLICATIONS, Physics Reports, vol. 329. P. 103-197

[11] LM. Pecora, TL. Carroll, (1990), "Synchronization in chaotic systems", Physics Rev Letters, vol. 64, p.821824 ,

[12] ET. Hunt, (1991), "Stabilizing high-period orbits in a chaotic system: The diode resonator", Physics Rev Letters, vol.66, p.1953-1991,

[13] Brown R, (1998), “Approximating the Mapping between Systems Exhibiting Generalized Synchronization”, Physics Rev Letter, vol. 81, p.4835,

[14] JZ. Yang, G. Hu, JH. Xiao, (1998), "Chaos Synchronization in Coupled Chaotic Oscillators with Multiple Positive Lyapunov Exponents”, Physics Rev Letters, vol. 80, pp. 496,

[15] EM. Shahverdiev, (2004), "Synchronization in systems with multiple time delays", Phys Rev E, vol. P. 70067202 ,

[16] Haque ME, Zhong LM, Rahman MF. (2003), A sensorless initial rotor position estimation scheme for a direct torque controlled interior permanent magnet synchronous motor drive. IEEETransPowerElectron; vol.18, p.1376-83.

[17] Li, Z., Park, J.B., Joo, Y.H., Zhang, B., Chen, G. Bifurcations and chaos in a permanent-magnet synchronous motor. IEEE Trans. Circuits Syst. I, Fundam. Theory Appl. 49, 383-387 (2002)

[18] Ge, X., Huang, J.: (2005) Chaos control of permanent magnet synchronous motor. In: Proc. of the Eighth International Conference on Electrical Machines and Systems, Nanjing, China, vol. 1, pp. 484-488

[19] Ren, H., Liu, D. (2006). Nonlinear feedback control of chaos in permanent magnet synchronous motor. IEEE Trans. Circuits Syst. II, Express Briefs 53, 45-50

[20] Ren, H., Liu, D., Li, J. (2003) Delay feedback control of chaos in permanent magnet synchronous motor. Proc Chin. Soc. Electron. Eng. Conf. Vol. 23, on. 6, p. 175178

[21] Harb, A.M.: Nonlinear chaos control in a permanent magnet reluctance machine. Chaos Solitons Fractals 19, 1217-1224 (2004)

[22] Wei, D., Luo, X., Wang, B., Fang, J. (2009), Robust adaptive dynamic surface control of chaos in permanent magnet synchronous motor. Phys. Lett. A 363, 71-77 (2007) Zribi, M., Oteafy, A., Smaoui, N.: Controlling chaos in the permanent magnet synchronous motor. Chaos Solitons Fractals 41(3), 1266-1276

[23] JANG, J.S.R. (1993). Anfis adaptive-network-based fuzzy inference system. Systems Man and Cybernetics, IEEE Transactions on, 23, 665-685

[24] WANG, Z., PALADE, V. \& XU, Y. (2006). Neurofuzzy ensemble approach for microarray cancer gene expression data analysis. In Evolving Fuzzy Systems, 2006 International Symposium on, $241-246$. 